\title{
High hydrostatic pressure extract of garlic increases the HDL cholesterol level via up- regulation of apolipoprotein A-I gene expression in rats fed a high-fat diet
}

Seohyun Lee ${ }^{1}$, Hyunjin Joo ${ }^{1}$, Chong-Tai Kim², In-Hwan Kim ${ }^{3}$ and Yangha Kim*

\begin{abstract}
Background: Cardiovascular disease (CVD) is the number one cause of mortality worldwide and a low high-density lipoprotein cholesterol (HDL-C) level is an important marker of CVD risk. Garlic (Allium sativum) has been widely used in the clinic for treatment of CVD and regulation of lipid metabolism. This study investigated the effects of a high hydrostatic pressure extract of garlic (HEG) on HDL-C level and regulation of hepatic apolipoprotein A-I (apoA-I) gene expression.

Methods: Male Sprague-Dawley rats were divided into two groups and maintained on a high-fat control diet $(\mathrm{CON})$ or high-fat control diet supplemented with high hydrostatic pressure extract of garlic (HEG) for 5 weeks. Changes in the expression of genes related to $\mathrm{HDL}-\mathrm{C}$ metabolism were analyzed in liver, together with biometric and blood parameters.

Results: In the HEG group, the plasma triglyceride (TG) and low-density lipoprotein cholesterol (LDL-C) levels were significantly decreased in comparison with the CON group $(P<0.05)$. Dietary HEG also lowered the hepatic TG and total cholesterol (TC) levels compared to the CON group. While the plasma HDL-C level and mRNA level of hepatic apoA-I, which is one of primarily proteins of HDL-C particle, were significantly increased in the HEG group compared to the CON group $(P<0.05)$. The gene expression of ATP-binding cassette transporter A1 (ABCA1) and lecithin:cholesterol acyltransferase (LCAT), importantly involved in the biogenesis in HDL, were also up-regulated by dietary HEG.
\end{abstract}

Conclusions: These results suggest that HEG ameliorates plasma lipid profiles and attenuates hepatic lipid accumulation in the high-fat fed rats. Our findings provides that the effects of HEG on the increase of the plasma $\mathrm{HDL}-\mathrm{C}$ level was at least partially mediated by up-regulation of hepatic genes expression such as apoA-I, ABCA1, and LCAT in rats fed a high-fat diet.

Keywords: Garlic, High hydrostatic pressure processing, HDL-cholesterol, Gene expression, Apolipoprotein A-I, ATP-binding cassette transporter A1, Lecithin:cholesterol acyltransferase

\footnotetext{
* Correspondence: yhmoon@ewha.ac.kr

${ }^{1}$ Department of Nutritional Science and Food Management, Ewha Womans University, Seoul, Republic of Korea

Full list of author information is available at the end of the article
} 


\section{Background}

Cardiovascular disease (CVD) is one of the major causes of mortality globally, and the number of deaths caused by CVD has been increased at an alarming rate [1]. Dyslipidemia, which includes high levels of blood total cholesterol (TC), low-density lipoprotein cholesterol (LDL-C), and triglyceride (TG), and low level of high-density lipoprotein cholesterol (HDL-C), is considered one of the major risk factors of CVD. Guidelines for the prevention of CVD have focused on decreasing LDL-C levels [2]. However, a substantial proportion of patients with CVD do not have elevated LDL-C levels [3] and the role of other lipoproteins, including HDL, in the progression of CVD and atherosclerosis has been recently investigated [3]. Several epidemiologic studies have reported clinical healthful benefits associated with raising HDL-C level and prevention of CVD [4-7]. Therefore, regulation of HDL-C level should be one of the main goals in the treatment of atherogenic dyslipidemia and reduction of the risk of CVD. Apolipoprotein A-I (apoA-I) is a major structural protein constituent in HDL particles, and plays an important role in regulating HDL biosynthesis [8]. Also, ATP-binding cassette transporter A1 (ABCA1) and lecithin:cholesterol acyltransferase (LCAT) directly contributes to HDL biogenesis [9].

Garlic (Allium sativum) is one of the most well-known herbal medicines worldwide and there has been increasing interest in using garlic as a cholesterol-lowering agent. However, the effect of garlic consumption on HDL-C level remains controversial and many studies have reported conflicting results regarding this relationship [10-12]. The major components of garlic are water, carbohydrates, proteins, fat, dietary fiber, and essential amino acids. It also contains high levels of saponins, vitamins, minerals, and various biologically active sulfur compounds such as alliin, allicin, ajoene, diallyl disulfide, allyl methanethiosulfinate, diallyltrisulfide, and S- allylcysteine [13,14]. However, these bioactive compounds of garlic are affected by processing methods; thus, the processing technique may be the main reason for the differences in the reported efficacy of various garlic preparations.

High hydrostatic pressure processing (HPP) is a method used to reduce microbial counts, and has been shown to affect enzyme activity and food product functionality since covalent bonds are not affected by pressure [15]. Thus, HPP may only minimally modify the nutritional value and sensory quality of fruits and vegetables. A recent study reported that HPP affected the content, category and intensity of the volatile compounds in garlic by decreasing the alliinase activity, which influenced the formation of allicin [16]. Furthermore, the effects of HPP on the total polyphenols, total flavonoid, antioxidant activities, and 5hydroxymethyl-2-furaldehyde (5-HMF) contents in garlic were previously examined [17]. The objective of this study was to elucidate the antidyslipidemic effect of the high hydrostatic pressure extract of garlic (HEG), produced by HPP, in rats fed a high-fat diet. This effect could be explained by alteration of the expression of genes that is involved in cholesterol metabolism. We hypothesized that HEG may increase concentration of blood HDL-C level via induction of genes expression, related with HDL biogenesis, such as apoA-I, ABCA1, and LCAT in rats fed a high-fat diet.

\section{Results \\ Body weight and energy intake}

Rats were assigned randomly to two experimental groups and the initial body weight of the rats was not significantly different between the two groups (Table 1). Final body weight was also not significantly different between the CON and HEG groups (Figure 1A; Table 1). There were no significant differences in the amount of energy intake and energy efficiency ratio between groups over the experimental period (Table 1).

\section{Liver weight and plasma AST and ALT activities}

At the doses given, HEG did not cause a rise in plasma AST and ALT activities when compared with those of CON group (Table 2). In addition, the liver weight (Table 1) was unaffected by HEG treatment. These data indicate that the HEG was well tolerated by the rats (Table 2).

\section{Plasma lipid profiles}

The HEG group had significantly lower plasma concentrations of TG and LDL-C by $19 \%$ and $24 \%$, respectively, in comparison with the CON group (Table 2). The atherogenic index (AI) and the ratio of LDL-C to HDL-C, which are all significant risk factors of CVD, were also significantly decreased by $45 \%$ and $24 \%$, respectively, in the HEG group as compared to the CON group (Table 2).

Table 1 Effect of dietary HEG supplementation on physiological variables

\begin{tabular}{lcr}
\hline \multicolumn{1}{c}{ Variables } & \multicolumn{1}{c}{ CON } & \multicolumn{1}{c}{ HEG } \\
\hline Initial body weight (g) & $107.43 \pm 1.52$ & $108.04 \pm 1.51$ \\
Final body weight (g) & $321.40 \pm 6.25$ & $319.25 \pm 8.63$ \\
Food intake (g/day) & $17.58 \pm 0.41$ & $17.56 \pm 0.61$ \\
Energy intake (kcal/day) & $81.64 \pm 1.91$ & $80.29 \pm 2.78$ \\
Energy efficiency ratio (EER) ${ }^{1)}$ & $0.74 \pm 0.00$ & $0.74 \pm 0.00$ \\
Liver weight & $3.01 \pm 0.06$ & $3.08 \pm 0.07$ \\
(g/100 g body weight) & & $1.28 \pm 0.07$ \\
Epididymal adipose tissue weight & $1.24 \pm 0.05$ & \\
(g/100 g body weight) & & \\
\hline
\end{tabular}

Values are expressed as mean $\pm S E M ; n=10$ for each treatment group.

1) Energy efficiency ratio $(E E R)=$ Body weight gain ( $g /$ day)/Energy intake (kcal/day). 

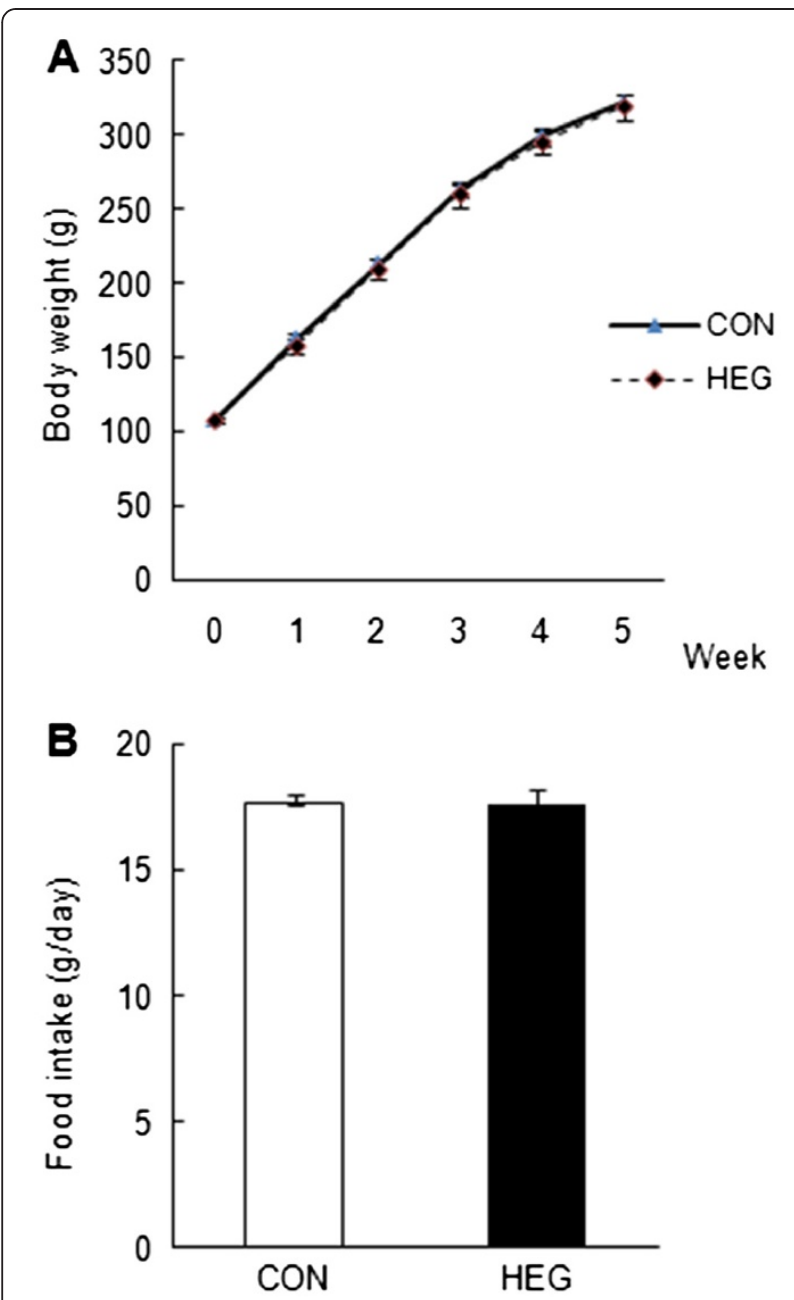

Figure 1 Effects of HEG supplementation on (A) body weight and (B) food intake. Body weight and food intake were measured twice a week. Values are expressed as mean \pm SEM $(n=10)$.

While the HDL-C level and the ratio of HDL-C to TC were significantly increased by $59 \%$ and $43 \%$, respectively, in the HEG group relative to the CON group in rats fed a high-fat diet (Table 2).

\section{Hepatic lipid profiles}

The contents of hepatic TG and TC were investigated to evaluate the effects of HEG on the reduction of hepatic lipids levels. The amounts of hepatic TC and TG were significantly reduced by $43 \%$ and $35 \%$, respectively, in the HEG group as compared to the CON group (Table 2). These results support the hypothesis that HEG may regulate TG and TC levels in rats fed a high-fat diet.

\section{Gene expressions of hepatic apoA-1, ABCA1, and LCAT}

ApoA-I is one of major structural protein constituents in HDL particle and alteration of the hepatic apoA-I gene expression affects blood apoA-I concentration.
Table 2 Effects of dietary HEG supplementation on plasma and hepatic lipid profiles

\begin{tabular}{lrr}
\hline & \multicolumn{1}{l}{ CON } & HEG \\
\hline Plasma & & \\
Total cholesterol (mg/dl) & $70.26 \pm 2.31$ & $68.57 \pm 4.60$ \\
LDL cholesterol (mg/dl) & $34.97 \pm 2.76$ & $26.49 \pm 3.40^{*}$ \\
HDL cholesterol (mg/dl) & $25.42 \pm 1.21$ & $40.35 \pm 6.30^{*}$ \\
Triglyceride (mg/dl) & $50.37 \pm 3.51$ & $40.64 \pm 3.94^{*}$ \\
LDL-C/HDL-C & $2.60 \pm 1.20$ & $1.97 \pm 0.26^{*}$ \\
HDL-C/TC & $0.37 \pm 0.02$ & $0.53 \pm 0.05^{*}$ \\
Atherogenic Index & $1.75 \pm 0.12$ & $0.97 \pm 0.18^{*}$ \\
AST (IU/L) & $65.45 \pm 8.29$ & $45.07 \pm 3.98^{*}$ \\
ALT (IU/L) & $9.07 \pm 0.79$ & $10.69 \pm 1.37$ \\
Hepatic lipids & & \\
Triglyceride (mg/g liver) & $6.46 \pm 0.65$ & $4.18 \pm 0.68^{*}$ \\
Total cholesterol (mg/g liver) & $1.70 \pm 0.09$ & $0.97 \pm 0.11^{*}$ \\
\hline
\end{tabular}

Values are expressed as mean $\pm S E M ; n=10$ for each treatment group. *: Significantly different from the CON group at $P<0.05$ by Student's $t$-test.

Thus, we investigated the effect of HEG supplementation on the mRNA level of apoA-I. Our data showed that the hepatic apoA-I mRNA level was 1.6- fold higher in the HEG group than in the CON group $(P<0.05)$ (Figure 2A). The mRNA levels of hepatic ABCA1 and LCAT, which are importantly involved in the HDL biogenesis, were also significantly increased by 1.5 - fold $(P<0.05)$ and 1.8 - fold $(P<0.01)$, respectively, in the HEG group as compared as the CON group (Figure 2B, C) in high-fat fed rats.

\section{Discussion}

We investigated the effects of HEG on the regulation of lipid profiles in rats fed a high-fat diet. The antilipidemic effects of garlic have been previously reported [18-20]. Our results showed that dietary HEG reduced hepatic TC and TG levels. Previous studies have reported that garlic reduces hepatic cholesterol and TG levels [21-23]. The hypocholesterolemic effects of the garlic extract stem occurred in part from the inhibition of hepatic cholesterol synthesis by water-soluble sulfur compounds, such as Sallylcysteine and ajoene [22]. Reduction of hepatic cholesterol may also be due to inhibition of cholesterol biosynthesis or activation of reverse cholesterol transport (RCT), which extracts cholesterol. Also, in vivo and in vitro studies have indicated that garlic and its constituents inhibit pivotal enzymes involved in the biosynthesis of cholesterol, such as HMG-CoA reductase [9,18,22].

In the present study, treatment with HEG reduced plasma TG concentration in rats fed a high-fat diet. This observation is in agreement with human [23,24] and animal [18] studies, which showed an inverse association between dietary garlic and the concentration of plasma TG. 


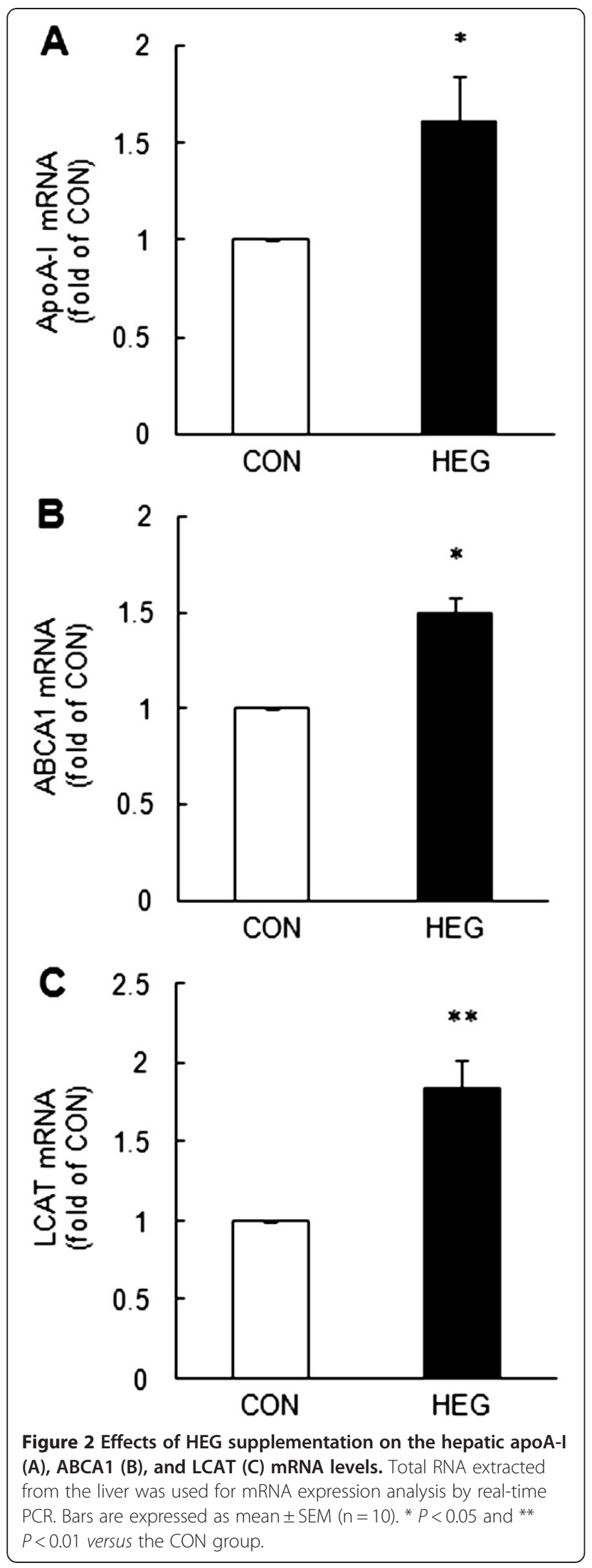

Also, studies using cell lines and animal models have shown that garlic has beneficial effects on reducing the accumulation of TG in blood. These effects were suggested with activation of liver phosphatidate phosphohydrolase (PAP) and fatty acid synthease (FAS), which play a critical role in controlling the biosynthesis of TG $[21,25,26]$.

Dietary HEG increased plasma HDL-C concentration and decreased plasma LDL-C concentration. The primary function of HDL is to remove cholesterol from peripheral cells and transport cholesterol to the liver for biliary excretion by RCT. In addition, HDL has been shown to prevent foam cell formation, thus retarding the progress of atherosclerosis [27]. Therefore, the present results clearly demonstrated that supplementation of HEG favorably modified cholesterol metabolism. Our findings were in agreement with other studies, which reported that garlic consumption increased HDLC level in hyperlipidemic individuals [10], animals [11], and patients with coronary artery disease [28]. The LDL$\mathrm{C} / \mathrm{HDL}-\mathrm{C}$ ratio and HDL-C/TC ratio are important indicators of CVD and atherosclerosis [29]. In this study, the $\mathrm{HDL}-\mathrm{C} / \mathrm{TC}$ ratio was higher and the LDL-C/HDL-C ratio was lower in the HEG group relative to the CON group. When garlic powder was added into the diet at a concentration of $1 \%$, plasma TC and LDL-C levels were decreased and HDL-C level were increased in rabbits [30], resulting in a decrease in the LDL-C/HDL-C ratio. Hence, our observations indicate that dietary HEG may have hypolipidemic effects by reducing the TC and TG levels in the body and CVD protection effects by regulating the HDL-C/TC and LDL-C/HDL-C ratios.

ApoA-I is a main protein component of HDL particles and constitutes about $70 \%$ of the apolipoprotein content of HDL particles [31], and is thought to be a better predictor of CVD risk than TC and LDL-C [32]. ApoA-I acts as a cofactor for LCAT, which is a key enzyme involved in removing excess cholesterol from tissues and incorporating it into the HDL [33]. Hence, plasma apoA-I concentration is correlated with plasma HDL-C concentration, and apoA-I mRNA level may be an important determinant of blood HDL-C concentration [34,35]. However, less is known about the effect of garlic on the HDL-C and the expression of genes associated with HDL-C metabolism.

Treatment with HEG increased the apoA-I mRNA level, which corresponded to an increase in the HDL-C level. Dorfman et al. [36] reported that treatment with dietary fatty acids, such as coconut oil and butter, resulted in an increase in both the blood HDL-C level and apoA-I concentrations, and these effects were found to be associated with the expression of apoA-I gene in animal studies. They indicate that regulation of apoA-I gene expression may be associated with the regulation of HDL-C level. Therefore, we suggest cautiously that 
dietary HEG supplementation may increase the HDL-C level with up-regulation of the apoA-I gene expression. Previous studies have shown that garlic consumption produces hypolipidemic effects without changing the blood HDL-C level [37]. Furthermore, although the apoA-I mRNA level is associated with HDL-C level, the increased level of HDL-C is not always caused by the change of hepatic apoA-I mRNA level. Choi et al. [38] reported that soy pinitol supplementation increased plasma HDL-C and apoA-I concentrations, whereas the expression of the hepatic apoA-I gene was not affected by soy pinitol. However, in our study, HEG supplementation was shown to increase the plasma HDL-C concentration with upregulation of apoA-I gene expression.

ApoA-I plays a pivotal role in RCT by functioning as an acceptor of cellular phospholipids and cholesterol and as an activator of LCAT. Recent studies indicated that the functional interactions of apoA-I with ABCA1 is necessary for the initial lipidation of apoA-I [9]. ABCA1 facilitates the efflux of cellular phospholipids and cholesterol to extracellular lipid-poor apoA-I, and lapidated apoA-I produces discoidal pre $\beta$-HDL particles. Thus, interaction between apoA-I and ABCA1 is an essential step for formation of HDL that ultimately determines plasma HDL-C level. The pre $\beta$-HDL particles are subsequently transformed into mature spherical HDL particles by LCAT, which is an enzyme that concerts free cholesterol into cholesteryl ester and is critical for the maintenance and maturation of HDL metabolism [9]. In this study, we have showed that the expressions of hepatic ABCA1 and LCAT were increased by HEG supplementation.

\section{Conclusions}

In conclusion, HEG ameliorated plasma lipid profiles by decreasing the LDL-C level and increasing the HDL-C level in high-fat fed rats. Also dietary supplementation with HEG attenuated hepatic lipid accumulation by reducing the contents of TG and TC. This study provides that the effects of HEG on the increase of the plasma HDL-C level was at least partially mediated by upregulation of genes related with HDL biogenesis, such as apoA-I, ABCA1, and LCAT. Accordingly, the antidyslipidemic effects of HEG imply that HEG has potential as a bioactive food ingredient for the prevention of CVD in rats fed a high-fat diet.

\section{Methods}

\section{Materials}

High hydrostatic pressure extract of garlic (HEG) was provided as a gift from the Korea Food Research Institute (Seongnam, Gyeonggi, Korea). The dietary HEG powder used in this study was prepared as follows: Cloves of fresh garlic were purchased from the local market (Seosan,
Chungnam, Korea). $550 \mathrm{~g}$ of the fresh garlic was crushed with $450 \mathrm{ml}$ of distilled water and digested with the following enzymes: Viscozyme L, Celluclast 1.5 L, and Liquozyme supra (Novo Nordisk, Bagsvaerd, Denmark). The garlic slurry was adjusted to $\mathrm{pH} 5.5$ and processed under $100 \mathrm{MPa}$ for a $24 \mathrm{hr}$ at $50^{\circ} \mathrm{C}$ using a high hydrostatic pressure machine TFS-2 L (Toyo Koatsu Co. Ltd., Hiroshima, Japan) to solubilize the garlic cell wall and to obtain the major component such as allicin and water soluble materials. The HEG contained $1.24 \mathrm{mg} / \mathrm{g}$ of allicin, which was higher than hot water extract of garlic $(0.86 \mathrm{mg} / \mathrm{g})$ as determined by high performance liquid chromatography. The hot water extract of garlic was prepared from the grounded garlic boiled in $100^{\circ} \mathrm{C}$ hot water for $1 \mathrm{hr}$. All other chemicals were of the highest pure grade available.

\section{Animals and diets}

Four-week-old male Sprague-Dawley (SD) rats (G-bio company, Gyeonggi, Korea), weighing $60 \sim 70$ g, were individually housed in temperature-controlled room $\left(22 \pm 2^{\circ} \mathrm{C}\right)$ under $12 \mathrm{hr}$ light/dark conditions. Rats consumed a commercial chow diet (Zeigler Bros Inc., PA, USA) and water ad libitum for a week to stabilize their metabolic condition. After acclimatization, the rats were completely randomized into two groups of ten rats each and maintained on one of the following diets: a high-fat control diet $(\mathrm{CON})$ or a high-fat control diet supplemented with $2 \%(\mathrm{w} / \mathrm{w})$ high hydrostatic pressure extract of garlic (HEG) for 5 weeks. To induce hyperlipidemia in the rats, they were maintained on a high-fat control diet (45\% of total energy as fat) based on the AIN-93 G diet with slight modifications [39] (Table 3). The amount of

Table 3 Composition of experimental diets ( $\mathrm{g} / \mathrm{kg}$ diet)

\begin{tabular}{lcr}
\hline \multicolumn{1}{c}{ Ingredient } & CON & HEG \\
\hline Casein & 170.73 & 170.73 \\
Sucrose & 121.95 & 121.95 \\
Corn Starch & 201.71 & 181.71 \\
Dyetrose & 155.00 & 155.00 \\
L-Cystine & 2.20 & 2.20 \\
Cellulose & 60.98 & 60.98 \\
Lard & 229.50 & 229.50 \\
Mineral Mix & & 42.68 \\
Vitamin Mix ${ }^{2)}$ & 42.68 & 12.20 \\
Choline Bitartrate & 12.20 & 3.05 \\
High hydrostatic pressure extract of garlic ${ }^{3)}$ & 3.05 & 20.00 \\
Fat\% (calories) & - & 45 \\
Total & 45 & 1000 \\
\hline 1) AIN-93 G mineral mixture. & 1000 & \\
2) AIN-93 G vitamin mixture. & & \\
3) The amount of corn starch in control diet (CON) was adjusted accordingly \\
when the dietary high hydrostatic pressure extract of garlic (HEG) was added.
\end{tabular}


corn starch in the CON was adjusted accordingly when the dietary HEG powder was added (Table 3). Food intake and body weight were measured twice a week during the treatment period (Monday, Thursday). The average weekly body weight and daily food intake of two groups were calculated. At the end of the experiment, the rats were deprived of food overnight and anesthetized with intramuscular injection of Zoletil/Rompun (Bayer, Leverkusen, Germany) mixture (4:1) at a dose of $0.1 \mathrm{ml} / 80 \mathrm{~g}$ body weight. A central longitudinal incision was made in the abdominal wall and blood samples were collected by cardiac puncture. Blood samples were centrifuged at $2800 \times g$ for $20 \mathrm{~min}$ at $4^{\circ} \mathrm{C}$ to obtain plasma, which was then stored at $-20^{\circ} \mathrm{C}$ for biochemical analyses. The liver was harvested, frozen immediately in liquid nitrogen, and stored in $-70^{\circ} \mathrm{C}$. All experimental protocols were approved by The Ewha Womans University Animal Experimentation Ethics Committee for the care and use of laboratory animals.

\section{Plasma biochemical measurements}

The plasma levels of TG, TC, HDL-C, AST and ALT were determined by the enzymatic colorimetric methods [40] using commercial kits (Asan Pharm Co., Ltd., Seoul, Korea). The Atherogenic Index (AI) was calculated using the formula of Rosenfeld [41]. In addition, the ratio of HDL-C to TC was calculated.

$$
* \mathrm{AI}=(\mathrm{TC}-\mathrm{HDL}-\mathrm{cholesterol}) / \mathrm{HDL}-\text { cholesterol }
$$

\section{Hepatic and fecal lipids analysis}

Hepatic and fecal lipids were extracted using the method described by Bligh and Dyer [42]. TC and TG were determined by enzymatic colorimetric methods using commercial kits as described above.

\section{Real-time qRT-PCR}

Total RNA was isolated from liver tissue using the Trizol reagent (Invitrogen, Carlsbud, CA, USA). The corresponding cDNA was synthesized from $4 \mu \mathrm{g}$ of total RNA using M-MLV reverse transcriptase. After cDNA synthesis, real-time qPCR was performed using universal SYBR Green PCR Master Mix on a fluorometric thermal cycler (Rotor-Gene 2000, Corbett Research, Mortlake, Australia). Primers were designed using an online program (Primer3; http://frodo.wi.mit.edu/) [43]. The sequences of the sense and antisense primers used for amplification were as follows: apoA-I, 5/-CTGGGTTCAACTGT TGGTCG-3/ and 5/-GGGCTGCATCTTCTGTTTCA3/; ABCA1, 5/- CCCAATCCCAAACACTCCTT-3/ and 5/-TCTTCATCGTCCAGTTCCCA-3/; LCAT, 5/- TAA CAATGGGTATGTGCGGG-3/ and 5/-GCCAAGGCTG TGTCCAATAA-3/; $\beta$-actin, 5/-GGCACCACACTTTCT
ACAAT-3 1 and 5/-AGGTCTCAAACATGATCTGG-3/. The delta delta $C_{t}$ method was used for relative quantification [44]. The delta $C_{t}$ value for each sample was determined by calculating the difference between the $C_{t}$ value of the target gene and the $C_{t}$ value of the $\beta$-actin reference gene. The normalized level of expression of the target gene in each sample was calculated using the formula $2^{-\Delta \Delta C t}$. Values were expressed as fold change over the control.

\section{Statistical analysis}

The effects of HEG on the plasma lipid concentrations and hepatic lipid contents were analyzed. All data are presented as mean \pm standard error of mean (SEM, $\mathrm{n}=$ number of rats). The statistical significances of the difference between the means of the two groups of samples (CON and HEG) were analyzed using Student's $t$-test. Statistical analyses were performed using the SAS software version 9.2 (SAS Inc., NC, USA). $P<0.05$ and $P<0.01$ were taken to indicate a significant difference.

\section{Abbreviations}

ABCA1: ATP-binding cassette transporter A1; Al: Atherogenic index; ApoAI: Apolipoprotein A-l; CON: High-fat control diet; CVD: Cardiovascular diseases; FAS: Fatty acid synthease; HDL-C: High-density lipoprotein cholesterol; HEG: High Hydrostatic pressure extract of garlic; HPP: High hydrostatic pressure processing; LCAT: Lecithin:cholesterol acyltransferase; LDL-C: Low-density lipoprotein cholesterol; PAP: Phosphatidate phosphohydrolase; RCT: Reverse cholesterol transport; TC: Total cholesterol; TG: Triglyceride.

\section{Competing interests}

The authors declare that they have no competing interests.

\section{Authors' contributions}

SL and YK conceived the study and its design. SL wrote the manuscript carried out all experiments, and analyzed data. HJ participated in the experiment for gene expression analysis. CK and IK contributed critical review, intellectual input in discussion and overall presentation of paper. YK refined the final draft and revised of this manuscript for publication. All authors read and approved the final manuscript.

\section{Acknowledgements}

The study was supported by the Food High Pressure Technology Development Project, Korea Food Research Institute (202007-03-03-WT011) and National Research Foundation of Korea (NRF-2010-0023066).

\section{Author details}

1 Department of Nutritional Science and Food Management, Ewha Womans University, Seoul, Republic of Korea. ${ }^{2}$ Food Bio-Nano Research Group, Korea Food Research Institute, Seongnam, Gyeonggi, Republic of Korea.

${ }^{3}$ Department of Food and Nutrition, College of Health Science, Korea University, Seoul, Republic of Korea.

Received: 29 February 2012 Accepted: 9 June 2012

Published: 19 June 2012

\section{References}

1. Deaton C, Froelicher ES, Wu LH, Ho C, Shishani K, Jaarsma T: The global burden of cardiovascular disease. J CardiovasC Nurs 2011, 26:S5-S14.

2. Expert Panel on Detection Evaluation and Treatment of High Blood Cholesterol in Adults: Third report of the National Cholesterol Education Program (NCEP) Expert Panel on Detection, Evaluation, and Treatment of High Blood Cholesterol in Adults (Adults Treatment Panel III) Final report. 2001. 
3. Kannel WB: Range of serum cholesterol values in the population developing coronary artery disease. Am J Cordiol 1995, 76:69C-77C.

4. Gordon T, Castelli WP, Hjortland MC, Kannel WB, Dawber TR: High density lipoprotein as a protective factor against coronary heart disease. The Framingham Study. Am J Med 1977, 62:707-714

5. Castelli WP, Garrison RJ, Wilson PW, Abbott RD, Kalousdian S, Kannel WB: Incidence of coronary heart disease and lipoprotein cholesterol levels. The Framingham Study. JAMA 1986, 256:2835-2838.

6. Assmann G, Schulte $H$, von Eckardstein A, Huang Y: High-density lipoprotein cholesterol as a predictor of coronary heart disease risk. The PROCAM experience and pathophysiological implications for reverse cholesterol transport. Atherosclerosis 1996, 124:S11-S20.

7. Sharrett AR, Ballantyne CM, Coady SA, Heiss G, Sorlie PD, Catellier D, Patsch W: Coronary heart disease prediction from lipoprotein cholesterol levels, triglycerides, lipoprotein(a), apolipoproteins A-I and B, and HDL density subfractions: the atherosclerosis Risk in Communities (ARIC) Study. Circulation 2001, 104:1108-1113.

8. Asztalos BF: High-density lipoprotein particles, coronary heart disease, and niacin. J Clin Lipidol 2010, 4:405-410.

9. Zannis VI, Chroni A, Krieger M: Role of apoA-I, ABCA1, LCAT, and SR-Bi in the biogenesis of HDL. J Mol Med 2006, 84:276-294.

10. Mahmoodi M, Islami MR, Asadi Karam GR, Khaksari M, Sahebghadam Lotfi A, Hajizadeh MR, Mirzaee MR: Study of the effects of raw garlic consumption on the level of lipids and other blood biochemical factors in hyperlipidemic individuals. Pak J Pharm Sci 2006, 19:295-298.

11. Thomson M, Al-Qattan KK, Bordia T, Ali M: Including garlic in the diet may help lower blood glucose, cholesterol, and triglycerides. J Nutr 2006, 136:800S-802S

12. Yeh YY, Yeh SM: Garlic reduced plasma lipids by inhibiting hepatic cholesterol and triacylglycerol synthesis. Lipids 1994, 29:189-193.

13. Kerckhoffs DA, Brouns F, Hornstra G, Mensink RP: Effects of the human serum lipoprotein profile of beta-glucan, soy protein and isoflavones, plant sterols and stanols, garlic and tocotrienols. J Nutr 2002, 132: 2494-2505.

14. Lawson LD, Wang ZJ: Low allicin release from garlic supplements: a major problem due to the sensitivities of alliinase activity. J Agric Food Chem 2001, 49:2592-2599.

15. Tauscher B: Pasteurization of food by hydrostatic high pressure: chemical aspects. Zeitschrift für Lebensmitteluntersuchung und Forschung 1995, 200: $3-13$

16. Ma Y, Song D, Wang Z, Jiang J, Jiang T, Cui F, Xiaobo F: Effect of ultrahigh pressure treatment on volatile compounds in garlic. Journal of food process engineering 2010, 34:1915-1930.

17. Kwon OC, Woo KS, Kim TM, Kim DJ, Hong JT, Jeong HS: Physicochemical characteristics of garlic (Allium sativum L) on the high temperature and pressure treatment. Korean J Food Sci Technol 2006, 38:331-336.

18. Yeh YY, Liu L: Cholesterol-lowering effect of garlic extracts and organosulfur compound: human and animal studies. J Nutr 2001, 131:989S-993S

19. Stevinson C, Pittler MH, Ernst E: Garlic for treating hypercholesterolemia: a meta-analysis of randomized clinical trials. Ann Intern Med 2000, 133: 420-429.

20. Koseoglu M, Isleten F, Atay A, Kaplan YC: Effects of acute and subacute garlic supplement administration on serum total antioxidant capacity and lipid parameters in healthy volunteers. Phytother Res 2010, 24: 374-378.

21. Lee M, Kim I, Kim C, Kim Y: Reduction of body weight by dietary garlic is associated with increase in uncoupling protein mRNA expression and activation of AMP-Activated protein kinase in diet-induced obese mice. J Nutr 2011, 141:1947-1953.

22. Gebhardt R, Beck H, Wagner KG: Inhibition of cholesterol biosynthesis by allicin and ajoene in rat hepatocytes and HepG2 cells. Biochim Biophys Acta 1994, 1213:57-62.

23. Silagy $C$, Neil A: Garlic as a lipid lowering agent-a meta-analysis. J R Coll Physicians Lond 1994, 28:39-45.

24. Warshafsky S, Kamer RS, Sivak SL: Effect of garlic on total serum cholesterol. A meta-analysis. Ann Intern Med 1993, 119:599-605.

25. Ali M, Al-Qattan KK, Al-Enezi F, Khanafer RM, Mustafa T: Effect of allicin from garlic powder on serum lipids and blood pressure in rats fed high fat cholesterol diet. Prostaglandins Leukot Essent Fatty Acids 2000, 62:253-259.
26. Heidarian E, Jafari-Dehkordi E, Seidkhani-Nahal A: Effect of garlic on liver phosphatidate phosphohydrolase and plasma lipid levels in hyperlipidemic rats. Food Chem Toxicol 2011, 49:1110-1114.

27. Turley SD, Dietschy JM: The liver: Biology and Pathobiology. New York: Raven Press; 1998:617-641.

28. Bordia A, Verma SK, Srivastava KC: Effect of garlic (Allium sativum) on blood lipids, blood sugar, fibrinogen and fibrinolytic activity in patients with coronary artery disease. Prostaglandins Leukotrienes Essent. Fatty acids 1998, 58:257-263.

29. Fernandez ML, Webb D: The LDL to HDL cholesterol ratio as a valuable tool to evaluate coronary heart disease risk. J Am Coll Nutr 2008, 27:1-5.

30. Kwon MJ, Song YS, Choi MS, Park SJ, Jeong KS, Song YO: Cholesteryl ester transfer protein activity and atherogenic parameters in rabbits supplemented with cholesterol and garlic powder. Life Sci 2003, 72: 2953-2964.

31. Lewis GF, Rader DJ: New insight into the regulation of HDL metabolism and reverse cholesterol transport. Circ Res 2005, 96:1221-1232.

32. Walldius G, Jungner I, Holme I, Asatveit AH, Kolar W, Steiner E: High apolipoprotein B, Low apolipoprotein A-I, and improvement in the prediction of fetal myocardial infarction (AMORIS study): a prospective study. Lancet 2001, 358:2026-2033.

33. Phillips MC, Gillotte KL, Haynes MP, Johnson WJ, Lund-Katz S, Rothblat GH: Mechanism of high density lipoprotein-mediated efflux of cholesterol from cell plasma membrane. Atherosclerosis 1998, 137:S13-S17.

34. Azrolan N, Odaka H, Breslow JL, Fisher EA: Dietary fat elevates hepatic apoA-I production by increasing the fraction of apolipoprotein A-I mRNA in the translating pool. J Biol Chem 1995, 270:19833-19838.

35. Ahn YS, Smith D, Osada J, Li Z, Schaefer EJ, Ordovas JM: Dietary fat saturation affects apolipoprotein gene expression and high density lipoprotein size distribution in Golden Syrian hamsters. J Nutr 1994, 124:2147-2155.

36. Dorfman SE, Wang S, Vega-López S, Jauhiainen M, Lichtenstein AH: Dietary fatty acids and cholesterol differentially modulate HDL cholesterol metabolism in Golden-Syrian hamsters. J Nutr 2005, 135:492-499.

37. Ackermann RT, Mulrow CD, Ramirez G, Gardner CD, Morbidoni L, Lawrence VA: Garlic shows promise for improving some cardiovascular risk factors. Arch Intern Med 2001, 161:813-824.

38. Choi MS, Lee MK, Jung UJ, Kim HJ, Do GM, Park YB, Jeon SM: Metabolic response of soy pinitol on lipid-lowering, antioxidant and hepatoprotective action in hamsters fed-high fat and high cholestero diet. Mol Nutr Food Res 2009, 53:751-759.

39. Reeves PG: Components of the AIN-93 diets as improvements in the AIN76A diet. J Nutr 1997, 127:S838-S841.

40. Omodeo Salè F, Marchesini S, Fishman PH, Berra B: A sensitive enzymatic assay for determination of cholesterol in lipid extracts. Anal Biochem 1984, 142:347-350.

41. Rosenfeld L: Lipoprotein analysis. Early methods in the diagnosis of atherosclerosis. Arch Pathol Lab Med 1989, 113:1101-1110.

42. Bligh EG, Dyer WJ: A rapid method of total lipid extraction and purification. Can J Biochem Physiol 1959, 37:911-917.

43. Rozen S, Skaletsky HJ: Primer3 on the WWW for general users and for biologist programmers. In Bioinformatics methods and protocols: methods in molecular biology. Edited by Krawetz S, Misener S. Totowa (NJ): Humana Press; 2000:365-386.

44. Livak KJ, Schmittgent TD: Analysis of relative gene expression data using real-time quantitative PCR and the $2^{-\nabla \nabla C T}$ method. Methods 2001 25:402-408

doi:10.1186/1476-511X-11-77

Cite this article as: Lee et al:: High hydrostatic pressure extract of garlic increases the HDL cholesterol level via up-regulation of apolipoprotein A-I gene expression in rats fed a high-fat diet. Lipids in Health and Disease 2012 11:77 